\title{
Ultrasound-Guided Cannulation of the Great Saphenous Vein in Neonates: A Randomized Study
}

\author{
Zhenzhen Tu, MD ${ }^{1} \quad$ Yanzhe Tan, MD ${ }^{1} \quad$ Lifei Liu, MD ${ }^{1} \quad$ jia Xie ${ }^{2} \quad$ Ying Xu, MD ${ }^{1}$ Wei Liu, MD ${ }^{1}$ \\ 1 Department of Anesthesiology, Ministry of Education Key
Laboratory of Child Development and Disorders, National Clinical
Research Center for Child Health and Disorders, China International
Science and Technology Cooperation Base of Child Development
and Critical Disorders, Children's Hospital of Chongqing Medical
University, Chongqing, P.R. China
2 Department of Neonatal Nursing, Children's Hospital of Chongqing
Medical University, Chongqing, P.R. China

\begin{abstract}
Address for correspondence Wei Liu, MD, Department of Anesthesiology, Ministry of Education Key Laboratory of Child Development and Disorders, National Clinical Research Center for Child Health and Disorders, China International Science and Technology Cooperation Base of Child Development and Critical Disorders, Children's Hospital of Chongqing Medical University, Chongqing 400010, P.R. China (e-mail: faithrefresh@126.com).
\end{abstract}

Am J Perinatol

\begin{abstract}
Keywords

- ultrasound guided

- epicutaneo-caval catheters

- neonates

- cannulation

- great saphenous vein

Objective This prospective randomized controlled study aimed to compare the ultrasound-guided (USG) technique with the standard single-wall puncture technique for epicutaneo-caval catheter (ECC) placement in neonates.

Study Design A total of 100 neonates were included in this study. All enrolled neonates were randomly divided into two groups $(n=50)$ : the USG group and the control group. The control group underwent standard single-wall puncture for ECC placement procedures, and the USG group underwent USG ECC placement procedures. Results The first attempt success rates (62 vs. $38 \% ; p=0.016$ ) and the total success rates ( 92 vs. $74 \% ; p=0.017$ ) were higher in the USG group than in the control group. The procedure time was shorter in the USG group than in the control group: 351.43 (112.95) versus 739.78 seconds (369.13), $p<0.001$. The incidence of adverse events was not significantly different between the two groups.

Conclusion Compared with the standard single-wall puncture method, USG cannulation is superior for neonatal ECC placement, with a higher success rate, and decreases the total procedural time.
\end{abstract}

Key Points

- Establishing ECCS in neonates is challenging and lead to multiple attempts and adverse events.

- Information on the efficiency of USG dynamic needle tip positioning for ECCs in neonates is lacking.

- Compared with the standard puncture method, USG cannulation is superior for neonatal ECC placement.

During perioperative and postoperative procedures, reliable intravascular access is a life-saving route for intravenous fluid therapy, antibiotic administration, and parenteral nutrition in critically ill neonates. Epicutaneo-caval catheters (ECCs) are often used as an alternative to central venous catheters given their safety in the context of prolonged use. ${ }^{1-3}$ However, received

April 9, 2021

accepted after revision

July 2, 2021
DOI https://doi.org/

10.1055/s-0041-1733958.

ISSN 0735-1631.

\footnotetext{
(C) 2021. The Author(s).

This is an open access article published by Thieme under the terms of the Creative Commons Attribution-NonDerivative-NonCommercial-License, permitting copying and reproduction so long as the original work is given appropriate credit. Contents may not be used for commercial purposes, or adapted, remixed, transformed or built upon. (https://creativecommons.org/ licenses/by-nc-nd/4.0/)

Thieme Medical Publishers, Inc., 333 Seventh Avenue, 18th Floor, New York, NY 10001, USA
} 
establishing ECCs in these patients is challenging given their small vascular diameters and very low weight., ${ }^{4,5}$ These challenges can lead to multiple attempts and adverse events, such as local tissue damage and thrombosis.

A recently reported ultrasound-guided (USG) cannulation technique called dynamic needle tip positioning allows for accurate visualization of the needle tip by alternately advancing the needle and ultrasound probe using the shortaxis out-of-plane approach. ${ }^{6}$ This technique has shown obvious advantages in pediatric peripherally inserted central catheters (PICCs). ${ }^{7}$ Inserting a vascular catheter is considered a more challenging procedure in neonates compared with children, and information on the efficiency of USG dynamic needle tip positioning for ECCs in neonates is lacking.

Our team modified the steps in the previously established dynamic needle tip positioning technique, and using this technique, we succeeded in guiding radial artery cannulation and internal jugular vein cannulation in neonates. ${ }^{8,9}$ Hence, we designed a randomized controlled trial to investigate the efficacy of ultrasound guidance for ECC placement in neonates. We hypothesized that accurate visualization of the needle tip with the USG technique would be superior to standard single-wall puncture for ECC placement in this challenging population.

\section{Materials and Methods}

The current prospective, random, and observational study was approved by the Institutional Review Board of Children's Hospital, Chongqing Medical University, Chongqing, China, in September 2019 and was registered in the Chinese Clinical Trial Registry (ChiCTRIOR-2000029724). Written informed consent was obtained from the parents of all included children.

We enrolled neonates ( $<28$ days after birth) scheduled to undergo total intravenous fluid therapy or parenteral nutrition between February 2020 and June 2020 at the Children's

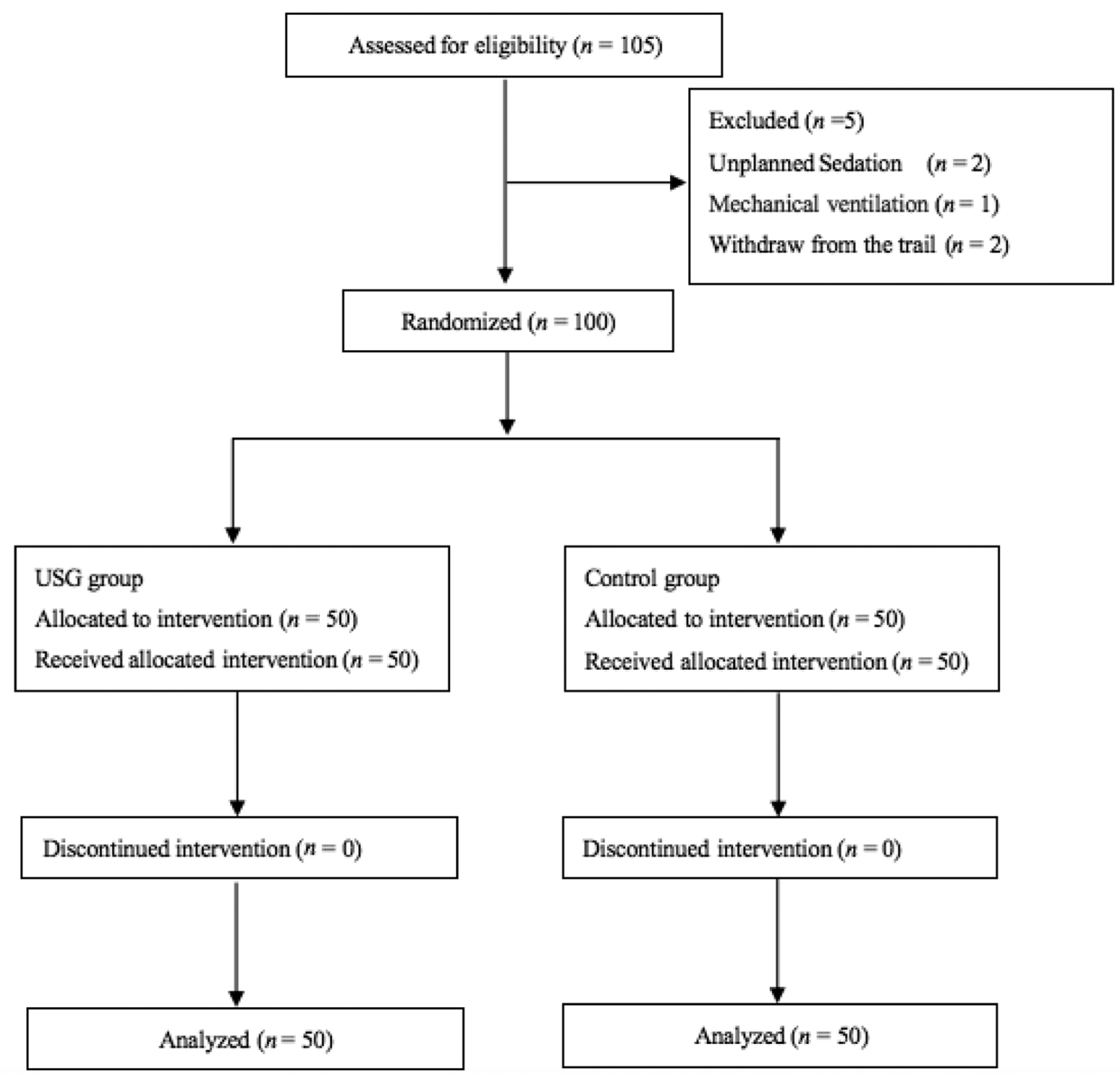

Fig. 1 The flow diagram according to the consolidated standards of randomized controlled trials. 
Hospital of Chongqing Medical University, Chongqing, China. Patients with signs of skin erosions, hematomas, or bone fracture near the insertion site or visible recent catheterization scars were excluded. In the neonatal intensive care unit (NICU), electrocardiography, peripheral capillary oxygen saturation, and noninvasive blood pressure (BP) were monitored. In these cases, the great saphenous vein (GSV) was punctured by using the USG technique or the standard single-wall puncture technique. If the catheterization fails for three times, it is defined as unsuccessful. All patients were followed for 7 days to ensure adequate catheter function and for potential complications associated with the catheter (-Fig. 1).

Using the random number method, enrolled neonates were randomly assigned to one of two groups: the USG group, in which ECC placement was performed by using the USG technique, or the control group, in which ECC placement was performed by using the standard single wall puncture method through direct visualization and palpation of the vein.

We used an USG ECC placement approach in the USG group using an ultrasonograph (GE Company, Loggie, Wuxi, Jiangsu, China) with a high-frequency probe $(10-22 \mathrm{MHz})$ and a PI catheter kit (1.9 Fr, Med Comp, Harleysville, Pennsylvania, USA). The procedures in the USG group were performed by the same operator who had performed more than 200 USG blood vessel catheterizations in neonates and infants; the procedures in the control group were performed by the same operator who had performed the standard single wall puncture method for ECCs in more than 200 neonates. All catheterizations were conducted in the GSV in the lower limb above the popliteal fossa. With the lower limb abducted and a tourniquet applied to the proximal thigh, the puncture site was disinfected and draped. The distance between the skin and GSV as well as vein diameter were measured with the same probe.

In the USG group, the USG technique was adopted for puncture. Specifically, the GSV was placed under the central arrow of the ultrasound image, and the needle was inserted into the middle of the ultrasonic probe (-Fig. 2A). The puncture needle remained motionless after entering the skin. The probe was moved 2 to $3 \mathrm{~mm}$ toward the proximal end and adjusted with the GSV in the ultrasonic image centered at the lower arrow and the needle positioned at the midpoint of the ultrasonic probe. The needle could be observed beyond the ultrasonic plane ( - Fig. 2B). Then, the ultrasonic probe was successively moved 2 to $3 \mathrm{~mm}$ straight toward the proximal end until it disappeared from the ultrasound image (-Fig. 2C). Then, the needle was shifted again until 2 to $3 \mathrm{~mm}$ of the tip appeared in the image (-Fig. 2D). Based on the relationship of the needle and the blood vessel, the direction of the needle was adjusted toward the center of the blood vessel such that the needle eventually pierced through the blood vessel. This procedure was repeated until the needle with the outer catheter was inserted as far as possible into the GSV. After placing the outer catheter into the vein as much as possible, the needle core was extracted. Then, the tourniquet was released, and the ECC wire was placed through it. Finally, the outer cannula was peeled away.

In the control group, ECC placement was performed by using the standard single wall puncture method through direct vein visualization and palpation of the vein. The needle was inserted at the mark until the needle core was filled with blood. Then, the outer cannula was slightly

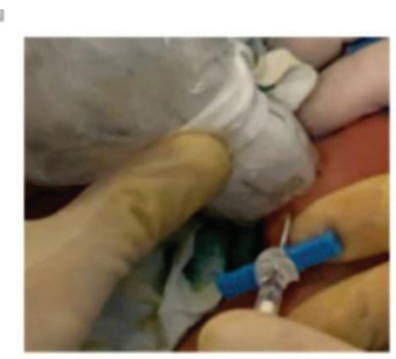

A-1

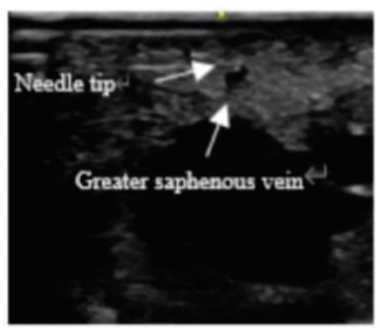

A-2

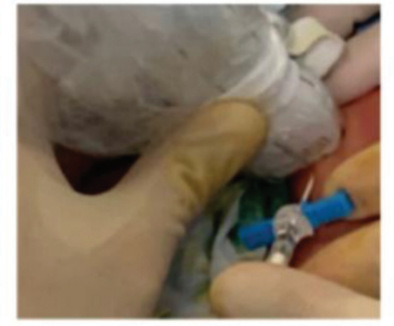

B-1

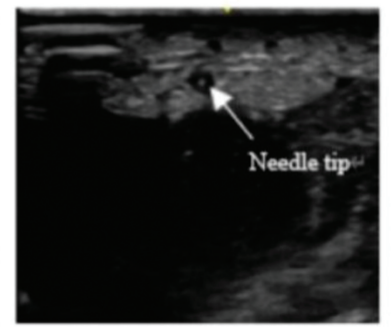

B-2

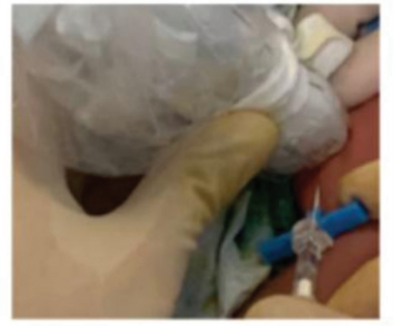

C-1

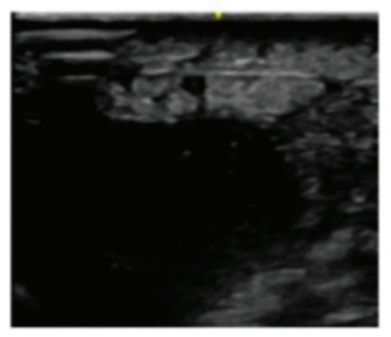

C-2

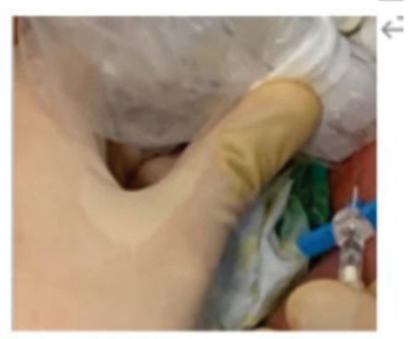

D-1

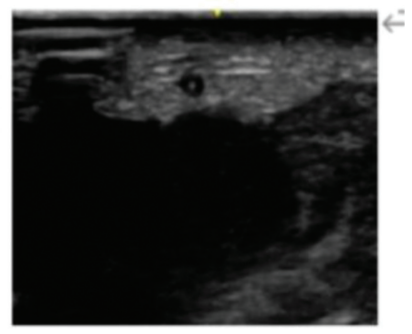

D-2

Fig. 2 The procedure of ultrasound-guided emergency cardiovascular care placement in neonates. The probe is moved to place the greater saphenous vein at the center of the ultrasound screen under the middle mark of the ultrasonic probe. The needle is inserted at the point at which the middle mark on the probe contacts the skin, and the needle tip, which is distinguished from the surrounding tissue, is displayed as a hyperechoic dot on the monitor (A). The needle is advanced toward the greater saphenous vein and inserted into the center of the vessel (B). The ultrasonic probe is successively moved toward the proximal end 2 to $3 \mathrm{~mm}$ straight to pinpoint disappears from the ultrasound images (C). The needle is advanced again until the tip appears in the image, based on the relationship of the needle and the blood vessels, and the direction of the needle is adjusted (D). 
advanced according to the operator's experience. The subsequent procedures were the same as those described above.

The insertion lengths of the ECC wire were premeasured by using a measuring tape from the insertion point to the 9 to 12 thoracic region, which was properly noted before starting the procedures. After ECC placement, the tip of the ECC wire was examined by chest X-ray.

The venous diameter was measured between the trailing and leading edges of the vein in the short-axis plane on twodimensional imaging. In addition, the venous depth was defined as the distance between the transducer and the near edge of the vein on two-dimensional imaging. The procedure was deemed successful when the catheter was placed smoothly to the intended depth, and central positioning of the catheter was confirmed by X-ray. Each distinct skin puncture was defined as an attempt. The procedure time was defined as the time from which the skin was punctured by the needle to the time at which the catheter had been placed into the vessel. Complications, including local venous injury and thrombosis, were examined during postoperative week 1. Local venous injury was defined as visible or palpable swelling around the puncture or cannulation site after the procedure. Thrombosis was defined as a clot detected via a venous Doppler test. Gestational age at insertion, sex, weight, GSV diameter, and depth were also recorded. These variables were recorded by an intensivist who did not perform the procedures.

\section{Statistical Analysis}

All statistical analyses were performed according to the intention-to-treat protocol. A two-sample independent $t$-test was used to compare continuous normally distributed data, whereas the Chi-square test or Fisher's exact test was used to compare categorical variables between the USG group and the control group. All statistical analyses were performed by using SPSS 22.0 (SPSS Inc., Chicago, IL).

\section{Results}

We enrolled 105 neonates. One neonate was excluded due to mechanical ventilation, two due to unplanned sedation, and two due to withdrawal from the trial. The remaining 100 neonates were randomized to the USG group or the control group in a $1: 1$ ratio $(n=50$ each; - Fig. 1$)$.

All neonates required ECC placement for intravenous fluid, drug administration, or parenteral nutrition therapy. The neonates' characteristics are shown in -Table 1. No significant differences in any parameter were noted between the two groups.

The first attempt success rates in the USG group and in the control group were 62 versus 38\%, respectively $(p=0.016)$. The total success rates in the USG group and the control group were 92 versus $74 \%$, respectively $(p=0.017)$. The mean (standard deviation) procedure time was shorter in the USG group compared with the control group (351.43 [112.95] vs. 739.78 seconds [369.13], respectively; $p<0.001$; - Table 2 ).

\begin{tabular}{|c|c|c|}
\hline Characteristics & $\begin{array}{l}\text { USG group } \\
(n=50)\end{array}$ & $\begin{array}{l}\text { Control group } \\
(n=50)\end{array}$ \\
\hline $\operatorname{Sex}(M / F)$ & $28 / 22$ & $23 / 27$ \\
\hline $\begin{array}{l}\text { gestational age } \\
\text { at insertion (wk) }\end{array}$ & $33.1(2.6)$ & $32.3(2.2)$ \\
\hline Weight (kg) & $1.6(0.5)$ & $1.6(0.5)$ \\
\hline $\begin{array}{l}\text { Puncture site } \\
\text { (right/left) }\end{array}$ & $29 / 21$ & $22 / 28$ \\
\hline Vein depth (mm) & $2.0(0.2)$ & $2.0(0.2)$ \\
\hline Vein diameter (mm) & $0.8(0.1)$ & $0.7(0.1)$ \\
\hline
\end{tabular}

Abbreviations: F, female; M, male; USG, ultrasound guided. Note: Continuous variables are presented as the means (standard deviation); categorical variables are presented as counts.

The incidence of local venous injury was lower in the USG group than in the control group (5/50 (10\%) vs. $11 / 50$ (22\%), respectively; $p=0.1017)$. One case of thrombosis occurred in the control group on postoperative day 7. Total adverse events were more frequent in the USG group than in the control group (5/50 (10\%) vs. $12 / 50$ (24\%), respectively; $p=0.0624$ ), but the difference was not statistically significant ( - Table 2 ).

\section{Discussion}

This randomized controlled trial demonstrated that the USG maneuver improves the success rate of ECC placement at the first attempt in neonates. The total success rate of catheterization, the time required for successful ECC placement, and adverse events were superior in the USG group compared with the control group. In our study, we specifically selected ultrasonography with a super high-frequency probe $(10-22 \mathrm{MHz})$, which can result in better imaging of the superficial vasculature. Two conditions of our conclusion are noted: the operator can use this type of ultrasonic skillfully, and catheterization was performed in the GSV.

The USG technique is useful for peripheral artery and venous catheterization in adult and pediatric patients. ${ }^{10-18}$ USG PICC placement has been recently reported, and some studies have demonstrated its efficacy in pediatric patients. ${ }^{7,19,20}$ However, neonates are a special group with small vein diameters and loose vascular tissue. The use of vascular puncture catheters under USG techniques is considered more challenging in neonates compared with children. ${ }^{4,5}$ Abdeyazdan et al reported ultrasound guidance for PICC placement compared with conventional methods, and the difference was not significant. ${ }^{4}$ The reason for the difference in our results, compared with the results of the previous study, could be that the USG method in the previous study was different from the USG technique used here. In our study, the USG technique involved moving the ultrasound transducer along with the needle, and real-time visualization of the needle tip position was facilitated during the short axis outof-plane insertion technique. ${ }^{8,9}$ This technique can overcome 
Table 2 Outcomes of emergency cardiovascular care placement procedures

\begin{tabular}{|c|c|c|c|c|c|}
\hline & USG group & Control group & OR $(95 \% \mathrm{Cl})$ & Chi-square & $p$-Value \\
\hline First-attempt success rate, $n(\%)$ & $31(62)$ & $19(38)$ & $0.376(0.168-0.842)$ & 5.7600 & $0.016^{\mathrm{a}}$ \\
\hline Total success rate, $n(\%)$ & $46(92)$ & $37(74)$ & $0.248(0.074-0.823)$ & 5.7406 & $0.017^{\mathrm{a}}$ \\
\hline Successful PICCs placement time (s) & $351.43(112.95)$ & $739.78(369.13)$ & & & $<0.001^{\mathrm{c}}$ \\
\hline \multicolumn{6}{|l|}{ Adverse events } \\
\hline Local venous injury, $n$ (\%) & $5(10)$ & $11(22)$ & $2.538(0.811-7.943)$ & 2.6786 & $0.1017^{\mathrm{a}}$ \\
\hline Thrombosis, n (\%) & 0 & $1(2 \%)$ & & & $1^{\mathrm{b}}$ \\
\hline Total adverse events, $n(\%)$ & $5(10)$ & $12(24)$ & $2.842(0.919-8.790)$ & 3.4727 & $0.0624^{\mathrm{a}}$ \\
\hline
\end{tabular}

Abbreviations: $\mathrm{Cl}$, confidence interval; OR, odds ratio; PICC, peripherally inserted central catheter; USG, ultrasound guided.

apearson's Chi-square test.

bFisher's exact test.

'Student's t-test.

Note: Categorical variables are presented as counts (percentages). Continuous variables are presented as the means (standard deviations).

the difficulty of visualizing needle tip positioning and its advancement toward the center of the vein during the static ultrasound technique in that study. Marcus D's team used a dynamic needle tip positioning technique for USG PICC placement in neonates weighing $<1.5 \mathrm{~kg}^{5} \mathrm{~A}$ high success rate among anesthesiologists in our hospital was reported in our preliminary trial by using the USG technique for internal jugular vein cannulation in neonates. ${ }^{9}$ To a certain extent, these findings support the results of our study.

There are several challenges to treating neonates with small vein diameters and low weight in our study population, but the USG technique has some obvious advantages. First, the USG technique can overcome the difficulty of distinguishing the vein and needle tip and can accurately confirm their relationship. In the standard single wall puncture method, the location of the vein is determined through direct visualization and palpation; however, the veins of neonates are difficult to observe and touch. In addition, the position of the needle tip is confirmed through return blood based on the operator's experiences, which may cause frequent trials due to inaccurate positioning. Visual imaging with USG can avoid repeated puncture. Second, the advancement of the needle through the target vein without posterior wall puncture can be visualized by the USG technique. However, using the standard single wall puncture method, advancement of the needle toward the posterior wall puncture is judged by the operator's experiences. In fact, in the standard single wall puncture method, a key reason for failure is vein posterior wall puncture in neonates with small vein diameters. Third, the outer cannula and needle are advanced up to the lumen of the vessel for a substantial distance $(\sim 0.5-1 \mathrm{~cm})$ by using the USG technique, allowing the ECC wire to pass easily. This procedure avoids the difficulty of the standard single wall puncture method in which the needle and outer cannula are only partially within the lumen, making it difficult for the ECC wire to pass easily into the lumen. We believe that the USG technique is effective in this study population.

Local venous injury is one of the puncture-related adverse events associated with ECC placement. In this study, the incidence of local venous injury was lower in the USG group compared with the control group given the increased firstattempt success rate in the USG group, which avoided repeated peripheral vein punctures. However, the difference was not significant, which could be due to the limited number of punctures (within three cases) in both groups. In addition, the incidence of thrombosis was consistent with the above result.

This study has some limitations. Using the USG technique for ECC placement in neonates is relatively straightforward. However, the experience level of the operators performing dynamic needle tip positioning may affect the results. In our study, the operator was sufficiently experienced in USG radial artery catheterization and internal jugular vein catheterization in neonates and infants, and had a high success rate in these patients. ${ }^{8,9}$ In addition, we analyzed only the GSV as the puncture site. Thus, this conclusion may not be applicable to other puncture sites. Therefore, the conclusion regarding other puncture points based on this method requires further study.

\section{Conclusion}

Compared with the standard single wall puncture method, improved visualization with the USG technique is superior for neonatal ECC placement with a higher success rate and shorter catheterization time. Therefore, this method is worthy of application in future clinical work.

\section{Authors' Contributions}

W.L. designed the trial and helped write the manuscript. Z.T., Y.T., and J.X. helped conduct the study. L.L. and Y.X. collected and analyzed the data.

\section{Funding}

This work was supported solely by departmental sources.

Conflict of Interest

None declared.

\section{Acknowledgments}

The authors would like to acknowledge the support of the staff from the Department of Anesthesiology and 
Department of Neonatal Nursing of Children's Hospital affiliated with Chongqing Medical University, for their cooperation in the study.

\section{References}

1 Li Z, Chen L. Comparison of ultrasound-guided modified Seldinger technique versus blind puncture for peripherally inserted central catheter: a meta-analysis of randomized controlled trials. Crit Care 2015;19:64

2 Molina Caballero AY, Martínez Merino M, Pérez Martínez A, Ayuso González L, Hernández Martín S, Pisón Chacón J. [Peripherally inserted central catheters: Savings, comfort, many advantages]. Cir Pediatr 2016;29(03):96-100

3 Yamaguchi RS, Noritomi DT, Degaspare NV, et al. Peripherally inserted central catheters are associated with lower risk of bloodstream infection compared with central venous catheters in paediatric intensive care patients: a propensity-adjusted analysis. Intensive Care Med 2017;43(08):1097-1104

4 Abdeyazdan Z, Sheikhan-Sudani E, Sadeghnia A, Talakoub S. Effect of using static ultrasound technique on peripherally inserted central catheters' insertion success rate in neonates in a neonatal intensive care unit. Iran J Nurs Midwifery Res 2014;19(06):643-646

5 Johnson KN, Thomas T, Grove J, Jarboe MD. Insertion of peripherally inserted central catheters in neonates less than $1.5 \mathrm{~kg}$ using ultrasound guidance. Pediatr Surg Int 2016;32(11):1053-1057

6 Clemmesen L, Knudsen L, Sloth E, Bendtsen T. Dynamic needle tip positioning - ultrasound guidance for peripheral vascular access. A randomized, controlled and blinded study in phantoms performed by ultrasound novices. Ultraschall Med 2012;33(07):E321-E325

7 Takeshita J, Inata Y, Ito Y, et al. Dynamic needle tip positioning for ultrasound-guided placement of a peripherally inserted central catheter in pediatric patients. J Cardiothorac Vasc Anesth 2020;34 (01):114-118

8 Liu L, Tan Y, Li S, Tian J. "Modified dynamic needle tip positioning" short-axis, out-of-plane, ultrasound-guided radial artery cannulation in neonates: a randomized controlled trial. Anesth Analg 2019;129(01):178-183

9 Tan Y, Tu Z, Ye P, et al. Ultrasound guidance for internal jugular vein cannulation in neonates: modified dynamic needle tip positioning technique versus longitudinal technique. J Vasc Access.. Doi: 10.1177/11297298211015043 2021
10 Thomas S, Moore CL. The vanishing target sign: confirmation of intraluminal needle position for ultrasound guided vascular access. Acad Emerg Med 2013;20(10):e17-e18

11 Hansen MA, Juhl-Olsen P, Thorn S, Frederiksen CA, Sloth E. Ultrasonography-guided radial artery catheterization is superior compared with the traditional palpation technique: a prospective, randomized, blinded, crossover study. Acta Anaesthesiol Scand 2014;58(04):446-452

12 Gopalasingam N, Hansen MA, Thorn S, Sloth E, Juhl-Olsen P. Ultrasound-guided radial artery catheterisation increases the success rate among anaesthesiology residents: a randomised study. J Vasc Access 2017;18(06):546-551

13 Gopalasingam N, Obad DS, Kristensen BS, et al. Ultrasoundguidance outperforms the palpation technique for peripheral venous catheterisation in anaesthetised toddlers: a randomised study. Acta Anaesthesiol Scand 2017;61(06):601-608

14 Kiberenge RK, Ueda K, Rosauer B. Ultrasound-guided dynamic needle tip positioning technique versus palpation technique for radial arterial cannulation in adult surgical patients: a randomized controlled trial. Anesth Analg 2018;126(01):120-126

15 Hanada S, Van Winkle MT, Subramani S, Ueda K. Dynamic ultrasound-guided short-axis needle tip navigation technique vs. landmark technique for difficult saphenous vein access in children: a randomised study. Anaesthesia 2017;72(12):1508-1515

16 Asheim P, Mostad U, Aadahl P. Ultrasound-guided central venous cannulation in infants and children. Acta Anaesthesiol Scand 2002;46(04):390-392

17 Takeshita J, Yoshida T, Nakajima Y, et al. Superiority of dynamic needle tip positioning for ultrasound-guided peripheral venous catheterization in patients younger than 2 years old: a randomized controlled trial. Pediatr Crit Care Med 2019;20(09):e410-e414

18 Takeshita J, Yoshida T, Nakajima Y, et al. Dynamic needle tip positioning for ultrasound-guided arterial catheterization in infants and small children with deep arteries: a randomized controlled trial. J Cardiothorac Vasc Anesth 2019;33(07):1919-1925

19 Subramanian S, Moe DC, Vo JN. Ultrasound-guided tunneled lower extremity peripherally inserted central catheter placement in infants. J Vasc Interv Radiol 2013;24(12):1910-1913

20 de Carvalho Onofre PS, da Luz Gonçalves Pedreira M, Peterlini MA. Placement of peripherally inserted central catheters in children guided by ultrasound: a prospective randomized, and controlled trial. Pediatr Crit Care Med 2012;13(05):e282-e287 